\title{
Molecular characterization of Gleason patterns 3 and 4 prostate cancer using reverse Warburg effect-associated genes
}

\author{
Ilinca Georgescu 1,2, Robert J. Gooding 2,3 , R. Christopher Doiron4, Andrew Day ${ }^{5}$, Shamini Selvarajah',
} Chris Davidson', David M. Berman ${ }^{1,2}$ and Paul C. Park ${ }^{1,6^{*}}$

\begin{abstract}
Background: Gleason scores (GS) $3+3$ and $3+4$ prostate cancers (PCa) differ greatly in their clinical courses, with Gleason pattern (GP) 4 representing a major independent risk factor for cancer progression. However, Gleason grade is not reliably ascertained by diagnostic biopsy, largely due to sampling inadequacies, subjectivity in the Gleason grading procedure, and a lack of more objective biomarker assays to stratify prostate cancer aggressiveness. In most aggressive cancer types, the tumor microenvironment exhibits a reciprocal pro-tumorigenic metabolic phenotype consistent with the reverse Warburg effect (RWE). The RWE can be viewed as a physiologic response to the epithelial phenotype that is independent of both the epithelial genotype and of direct tumor sampling. We hypothesize that differential expression of RWE-associated genes can be used to classify Gleason pattern, distinguishing GP3 from GP4 PCa foci.

Methods: Gene expression profiling was conducted on RNA extracted from laser-capture microdissected stromal tissue surrounding 20 GP3 and 21 GP4 cancer foci from PCa patients with GS $3+3$ and GS $\geq 4+3$, respectively. Genes were probed using a 102-gene NanoString probe set targeted towards biological processes associated with the RWE. Differentially expressed genes were identified from normalized data by univariate analysis. A top-scoring pair (TSP) analysis was completed on raw gene expression values. Genes were analyzed for enriched Gene Ontology (GO) biological processes and protein-protein interactions using STRING and GeneMANIA.
\end{abstract}

Results: Univariate analysis identified nine genes (FOXO1 (AUC: 0.884), GPD2, SPARC, HK2, COL1A2, ALDOA, MCT4, NRF2, and ATG5) that were differentially expressed between GP3 and GP4 stroma $(p<0.05)$. However, following correction for false discovery, only FOXO1 retained statistical significance at $q<0.05$. The TSP analysis identified a significant gene pair, namely ATG5/GLUT1. Greater expression of ATG5 relative to GLUT1 correctly classified $77.4 \%$ of GP3/GP4 samples. Enrichment for GO-biological processes revealed that catabolic glucose processes and oxidative stress response pathways were strongly associated with GP3 foci but not GP4. FOXO1 was identified as being a primary nodal protein.

Conclusions: We report that RWE-associated genes can be used to distinguish between GP3 and GP4 prostate cancers. Moreover, we find that the RWE response is downregulated in the stroma surrounding GP4, possibly via modulation of FOXO1.

Keywords: Prostate cancer, Gleason pattern, Reverse Warburg effect, Biomarkers, Gene expression, NanoString

\footnotetext{
*Correspondence: pcpark@gmail.com

1 Department of Pathology and Molecular Medicine, Queen's University,

Kingston, ON, Canada

6 Ontario Institute for Cancer Research, Toronto, ON, Canada

Full list of author information is available at the end of the article
} 


\section{Background}

In order to assess the risk of metastasis in prostate cancer (PCa), Gleason grading integrates the relative abundance of cancer cells that make low-grade patterns (Gleason pattern 3 or GP3) with those that make high-grade patterns (Gleason patterns 4 or 5 or GP4 and GP5). Cancers with more abundant high-grade patterns obtain higher Gleason scores (GS) and have higher risk of metastasis and death. Those with only low-grade cancer cells have almost no risk of PCa-specific death [1, 2]. GS exhibits a strong correlation with clinical outcome. However, scores are often misreported due to differences in grading performed by individual pathologists, and undersampling during biopsy, which occurs in up to $30 \%$ of cases [3]. Prognostic biomarkers are urgently needed to augment clinicopathologic parameters, such as GS, in the risk stratification of this disease. Unlike other epithelial tumors, such as breast tumors, PCa lacks confirmed molecular subtypes that differ in their prognosis or treatment response. Several biomarkers/panels that have been identified to date have thus far not been widely embraced by clinicians [4-11]. Notably, the gene features among these panels exhibit little overlap, reinforcing the notion of molecular heterogeneity in the progression of PCa [12-15].

Emerging evidence indicates that in many cancers, the tumor microenvironment plays a crucial role in their phenotypic progression [16-19]. Data from cell lines and animal models indicate that reciprocal interactions between cancer cells and cancer-associated fibroblasts (CAFs) facilitate growth and dissemination of tumor cells [20-24]. Central to this reciprocal relationship is the paradigm of altered glucose metabolism and metabolic coupling between cancer cells and CAFs known as the reverse Warburg effect (RWE) [25]. Specifically, cancer cells induce oxidative stress in adjacent stroma by promoting autophagy and lysosomal destruction of the mitochondria in CAFs, thereby diverting their favored metabolic pathway to aerobic glycolysis. The resulting high-energy by-products, such as lactate and ketones, are shuttled back to the tumor cells, in which they fuel increased oxidative phosphorylation and efficient ATP production needed for anabolic growth [26]. Oxidative stress also enhances the production of free radicals, resulting in increased DNA damage and random mutagenesis in cancer cells $[27,28]$. This relationship is maintained in most aggressive cancers and is indeed recognized as one of its emerging hallmarks.

Given that the changes experienced by the stroma are likely to be reflective of cancer growth and progression, probing the metabolic state of CAFs may provide a means of indirectly assessing the phenotypic state of the cancer cells, while bypassing the problem of epithelial genotypic heterogeneity and the need for direct sampling of the cancer cells themselves [29,30]. Additionally, because the tumor microenvironment is comprised of relatively benign cells, its genomic features are likely to be more reliable [31-34], making it an ideal medium in which to profile physiological responses, such as the RWE, to genetically dissimilar patterns of PCa.

Therefore, in this study, we compare the RWE status in the stromal component between aggressive and indolent PCa foci. Specifically, we compare the expression levels of 102 RWE-associated genes in the stroma adjacent to GP3 foci from GS $3+3$ tumors to those of GP4 foci from GS $\geq 4+3$ tumors. We report two potential classifiers that discriminate GP3 from GP4 tumor foci. Based on the clinical behavior and the histologic features of GS $3+3$ and GS $>4+3$ tumors, we propose potential roles for these genes in establishing their respective phenotypes.

\section{Methods}

\section{Human prostate tumor samples}

This study was conducted under the approval of the Research Ethics Board at Queen's University. The pathology database at the Kingston General Hospital was queried for radical prostatectomies between the years 2001 and 2013. Through the review of the pathology reports, suitable cases were identified for the two groups in our cohort. The first consisted of cases of organconfined diseases of GS $3+3$, with no evidence of higher tertiary pattern. The second group consisted of diseases with GS $\geq 4+3$, with or without evidence of extraprostatic involvement (Additional file 1: Table S1). Together, the GS $3+3$ and the GS $\geq 4+3$ groups represent divergent prognoses, "low-risk" and "intermediate to high-risk," respectively, for localized PCa [35]. Each of the selected cases were retrieved for histologic review by one of two urologic pathologists (DMB, CD) to confirm the diagnosis, according to the International Society of Urological Pathology (2005) Consensus guidelines [36]. In total, 20 GS $3+3$ samples and $21 \mathrm{GS} \geq 4+3$ samples were selected for this study.

\section{Sample processing}

GP3 foci and GP4 foci were identified from hemotoxylin and eosin-stained slides of GS $3+3$ and GS $>4+3$ samples, respectively. Corresponding archival formalinfixed paraffin-embedded (FFPE) blocks were sectioned and mounted on slides. Stromal tissue adjacent to cancer foci was harvested by laser capture microdissection, using a Zeiss PALM CombiSystem microscope. A minimum of $3 \times 106 \mu \mathrm{m}^{2}$ of stroma was harvested for each sample, using multiple serial sections, where necessary, to restrict the field of harvest to within ten cell widths from the margin of the epithelial foci.

RNA was extracted from microdissected tissue using the RNeasy $^{\odot}$ FFPE Kit (Qiagen, Valencia, CA, USA). The manufacturer's protocol was modified to substitute 
the proteinase digestion with that of Roche's PCRgrade recombinant Proteinase $\mathrm{K}$ (Roche Diagnostics Mannheim, Germany) at $56^{\circ} \mathrm{C}$ for $30 \mathrm{~min}(18.6 \mathrm{mg} / \mathrm{ml})$. The final elution step was conducted using RNase-free water heated to $37^{\circ} \mathrm{C}$ and repeated twice in order to increase yield. RNA was quantified using the Agilent RNA 6000 Pico Kit (Agilent Technologies, Santa Clara, CA, USA), following manufacturer's protocol, and stored at $-80^{\circ} \mathrm{C}$ until use. RNA quality was assessed by smear analysis and RNA integrity numbers (RIN) using the Agilent 2100 Bioanalyzer.

\section{Compilation of candidate RWE gene panel}

A gene panel representative of the RWE was generated using a three-pronged in silico approach. As a starting point, the literature was mined to identify genes with known associations to the RWE in both breast and prostate carcinomas [25, 27, 28, 37-42]. By design, genes from the primary list were grouped based on primary biological function into the following categories: hypoxia response/oxidative stress regulation, mitophagy, autophagy and mitochondrial dysfunction, glucose metabolism, myo-fibroblast differentiation and CAF markers, and metabolite transporters. These small groups of genes were then input into a network-building algorithm, STRING (http://stringdb.org), and nodal points that possessed a combined functional-evidence confidence score of greater than 0.9 , with more than three of the input genes, were noted.

Lastly, in order to further enrich the target gene list, the following Gene Omnibus (http://geneontology.org) [43] databases comparing the transcriptomes of lasercaptured microdissected or cultured stromal tissue derived from normal and invasive human breast and prostate carcinomas were accessed: GSE34312 [44], GSE26910 [45], and GSE11682 [46]. For each dataset, samples were assigned to either "normal-associated" or "tumor-associated" groups based on experimental labeling and compared using the GEO2R analysis software provided by the Gene Omnibus database (http://www. ncbi.nlm.nih.gov/geo/geo2r/). GEO2R generates a list of the top 250 differentially expressed genes, ranked by $p$ value. Genes with an adjusted $p<0.05$, an absolute value of the log base two fold change $>1.5$, and a biological function that fits into either of the above-mentioned categories were marked for inclusion. Genes identified through network building and Gene Omnibus database searches were subject to literature review prior to inclusion in the final gene panel. The final panel consisted of 102 target genes (Additional file 2: Table S2) associated with RWE for use in gene expression profiling. Five housekeeping genes were selected for inclusion, namely $A C T B, C L T C$, GUSB, HPRT1, and TUBB, as these genes had proven suitable for normalization in previous $\mathrm{PCa}$ gene expression profiling studies [47].

\section{cDNA conversion and multiplexed target enrichment for nCounter analysis}

Prior to hybridization, target enrichment was performed using a multiplexed target enrichment (MTE) protocol (NanoString Technologies, Seattle, WA, USA). Primer pairs were designed for each of the 102 target genes using Primer3 software. These primer pairs (Integrated DNA Technologies, Coralville, IA, USA) flanked the 100-nucleotide target regions specific to the NanoString probes (Additional file 3: Table S3). Two nanograms of RNA for each sample was reverse transcribed and amplified for 20 cycles, using SuperScript VILO MasterMix (Life Technologies, Carlsbad, CA, USA) and TaqMan ${ }^{\odot}$ PreAmp Master Mixes (Applied Biosystems, Foster City, CA, USA), respectively.

\section{Sample hybridization and $\mathrm{nCounter}$ analysis}

A digital multiplexed NanoString nCounter analysis (NanoString Technologies, Seattle, WA, USA) for gene expression was performed using $11 \mu \mathrm{L}$ of denatured amplified stromal cDNA. Each sample was probed against the custom RWE NanoString panel, which included 102 RWE-associated genes, 5 housekeeping genes, 6 spikedin positive controls ranging in concentration from 128 to $0.128 \mathrm{fM}$, and 8 synthetic negative control sequences (Additional file 4: Table S4). The hybridization reaction was prepared according to the nCounter Single Cell Expression Assay protocol (NanoString Technologies, Seattle, WA, USA). The digital analyzer pre-processed barcode images internally according to standard specifications for binding density and field of view (FOV). All samples were used, as their binding density was inside the recommended 0.05-2.25-range, and their percent FOV was greater than $75 / 280$.

\section{Processing and data normalization}

A protocol for NanoString gene expression data normalization was developed in-house based on the NanoStringNorm Bioconductor package [48]. Data normalization included positive control normalization, background correction, and sample content normalization Additional file 5: Figure S1 and Additional file 6: Figure S2. Our housekeeping collection was not used in normalization since only one gene, $A C T B$, showed reproducibility across all samples Additional file 7: Figure S3. (Within the NanoStringNorm package, the relevant options were CodeCount = "geo.mean," Background = "mean," SampleContent = "top.geo.mean.")

Briefly, the geometric mean of the six spiked-in positive controls taken across all lanes was divided by the geometric mean of each lane to create positive control 
correction factors for each sample. Positive normalization factors were accepted if they fell within the recommended range of $0.3-3$. Following positive control normalization, the mean of the negative control counts was used to estimate the background for each lane. This less conservative approach allowed for low expression profile distributions to be statistically probed. The sum of the geometric means for the top 75 genes with the highest intensities was divided by the sum of the top 75 genes within a single sample in order to produce a sample content normalization factor for each lane. The sample content correction factors were then multiplied against the control-normalized data. Sample content normalization factors were deemed acceptable if they ranged between the standard 0.1 and 10 . Prior to our final statistical analysis, those samples with $>50 \%$ missing data were excluded. Similarly, genes with $>50 \%$ missing data across samples were discarded.

\section{Statistical analysis}

Univariate gene expression differences were assessed using the rank-based non-parametric Mann-Whitney $U$ (MWU) test, appropriate for manifestly non-normal distributions, as well as the Welch $t$ test, noting the nonequivalence of sample variances. To account for small sample numbers, we employed both tests with a cutoff of $p=0.05$, being more confident in identifying expression profile differences if both the medians and means were found to be different. The Benjamini-Hochberg false discovery rate (FDR) correction [49], using the p.adjust routine from $\mathrm{R}$, was applied to both $p$ values to account for multiple comparisons, thereby producing a $q$ value for each test. Receiver operator curves (ROC) were generated for genes of interest in order to determine their accuracy in distinguishing GP3 from GP4.

Top-scoring pair (TSP) analysis [50] was used to identify pairs of genes that successfully classify GP3 from GP4. This method is employed to find a classifier that is not dependent on certain subjective decisions, specifically the calculation of sample content normalizations, or the setting of arbitrary cutoffs for normalization factors made when processing and normalizing the data, or the expression of reference genes. Here, prior to TSP analysis, raw data were mean-background corrected, followed by the exclusion of genes and then samples possessing $>50 \%$ zero values. Permutation testing was used to query the significance of the top-scoring pair under the null hypothesis that gene count is not associated with Gleason pattern. One hundred thousand random classification assignments were run to generate a score distribution.

\section{Pathway analysis}

Pathway analysis was conducted using STRING (http:// string-db.org) [51] and GeneMANIA (http://www. genemania.org) [52]. All genes that exhibited an area under the curve (AUC) greater than 0.7 in the ROC analysis were used as input. The gene list was then augmented with those having a $p<0.1$ for either test with a minimum fold change of 1 . In total, 17 upregulated genes and 4 downregulated genes, in GP3 relative to GP4, were used as inputs. Input genes were enriched for 5 and 10 related genes in STRING. Network significance, in all cases, was based on a GO [53] biological process-based weighting (http://geneontology.org) with correction for FDR. The STRING results were compared to those found using GeneMANIA software.

\section{Results}

\section{Assessing stromal FFPE sample quality}

All RNA samples had RINs ranging between 2 and 2.5, typical of fragmented RNA extracted from FFPE tissue [54]. Smear analysis revealed variability in the amount of fragmentation between samples, with the percentage of $50-300$ residue length fragments ranging between 50 and $84 \%$. Low yields and fragmentation of RNA necessitated RNA amplification prior to profiling.

\section{Processing and normalization of NanoString gene expression data}

All 41 samples passed binding density and FOV quality control measures implemented by NanoString. One sample (GP4-18) was removed during QC assessment due to its large normalization factor. Even with the application of the modestly conservative background correction, low counts resulted in a significant proportion of zero values within samples, as well as across samples for select genes. To eliminate unreliable data, seven samples which failed to register counts in $>50 \%$ of the genes (GP3-5, GP3-6, GP3-7, GP3-12, GP3-13, GP3-20, and GP4-12) were excluded from further analysis. Similarly, two genes (NOS2 and TKTL1) which failed to register counts in $>50 \%$ of the samples were also excluded from further analysis. Following data normalization and exclusion based on missing values, 98 genes and $33 \mathrm{PCa}$ samples (15 GP3, $18 \mathrm{GP} 4)$ passed all thresholds set for normalization and background correction and were included in downstream univariate analysis.

\section{Univariate analysis of differentially expressed genes associated with RWE}

Univariate statistical analysis was applied to normalized gene expression data, followed by in-house data processing techniques. In the present cohort, nine genes were differentially expressed between GP3 and GP4 stroma using both the MWU and Welch $t$ tests $(p=0.05)$. These genes (Table 1), listed in order of statistical significance, are FOXO1, GPD2, SPARC, HK2, COL1A2, ALDOA, SLC16A4 (MCT4), NRF2, and ATG5. Two additional genes, SIRT3 and ACTA2, were found to be significant by 
Table 1 Differential expression of RWE-associated genes in GP3 versus GP4 stroma

\begin{tabular}{llllll}
\hline Gene & $p($ Welch $t)$ & $p(\mathrm{MWU})$ & $q$ & ROC (AUC) & $\log _{2}(\overline{\mathrm{GP}} / \overline{\mathrm{GP}})$ \\
\hline FOXO1 & 0.0008 & 0.0005 & 0.0495 & 0.884 & -4.590 \\
GPD2 & 0.0046 & 0.0034 & - & 0.707 & -3.58 \\
SPARC & 0.0194 & 0.0119 & - & 0.769 & 3.12 \\
HK2 & 0.0172 & 0.0132 & - & 0.752 & -3.82 \\
COL1A2 & 0.0191 & 0.0200 & - & 0.742 & 2.16 \\
ALDOA & 0.0118 & 0.0231 & - & 0.778 & -2.60 \\
SLC16A4 & 0.0392 & 0.0327 & - & 0.750 & -3.43 \\
NRF2 & 0.0197 & 0.0349 & - & 0.729 & 2.46 \\
ATG5 & 0.0239 & 0.0410 & - & 0.748 & -2.79 \\
ACTA2 & 0.0255 & 0.1083 & - & - & 0.90 \\
SIRT3 & 0.0375 & 0.1505 & - & - & -3.25 \\
\hline
\end{tabular}

the Welch $t$ test but not by the MWU test. Seven of differentially expressed genes, FOXO1 GPD2, HK2, ALDOA, $S L C 16 A 4, A T G 5$, and SIRT3, were upregulated in the stroma from GP3 relative to GP4, while SPARC, COL1A2, $N R F 2$, and $A C T A 2$ were downregulated in GP3 stroma relative to GP4. Notably, FOXO1, SPARC, HK2, and MCT4 exhibited the highest magnitude of $\log _{2}$ fold changes of $4.58,3.12,3.81$, and 3.46 , respectively.

To account for multiple comparisons, a BenjaminiHochberg FDR correction was applied to the MWU test values using p.adjust from the Bioconductor $\mathrm{R}$ package. As expected, the FDR was very high given the small sample size compared to the number of variables. After correction for FDR, only one gene, FOXO1, retained statistical significance with an adjusted $p$ value of $q=0.0495$. A notched box-and-whisker plot for this gene's expression values is shown in Fig. 1.

To assess the suitability of the differentially expressed genes as classifiers, ROC curves were generated. FOXO1 produced an AUC of 0.884 (Fig. 2), suggesting a high discriminatory power between GP3 and GP4. The remaining eight genes had individual AUCs ranging between 0.71 and 0.78 . Notched box-and-whisker plots for these eight genes are shown in Fig. 3.

\section{Pathway analysis}

To establish signalling networks that may play significant biological roles in GP3 and GP4, subject to the provision that genes are taken only from our original RWE gene list, STRING pathway analysis software was employed using enrichment for the most meaningful GO biological processes. Irrespective of the use of all 21 genes, namely 17 upregulated genes (FOXO1, GPD2, HK2, MYC, ALDOA, MCT4, ATG5, TGFB2, TGFB3, EGLN1, GAPDH, CA9, P4HA1, MXI1, MMP9, and PGM1) and 4 downregulated genes (COL1A2, SPARC, NRF2, and TGFBR2) or only those 17 genes that are upregulated in GP3 relative to GP4,

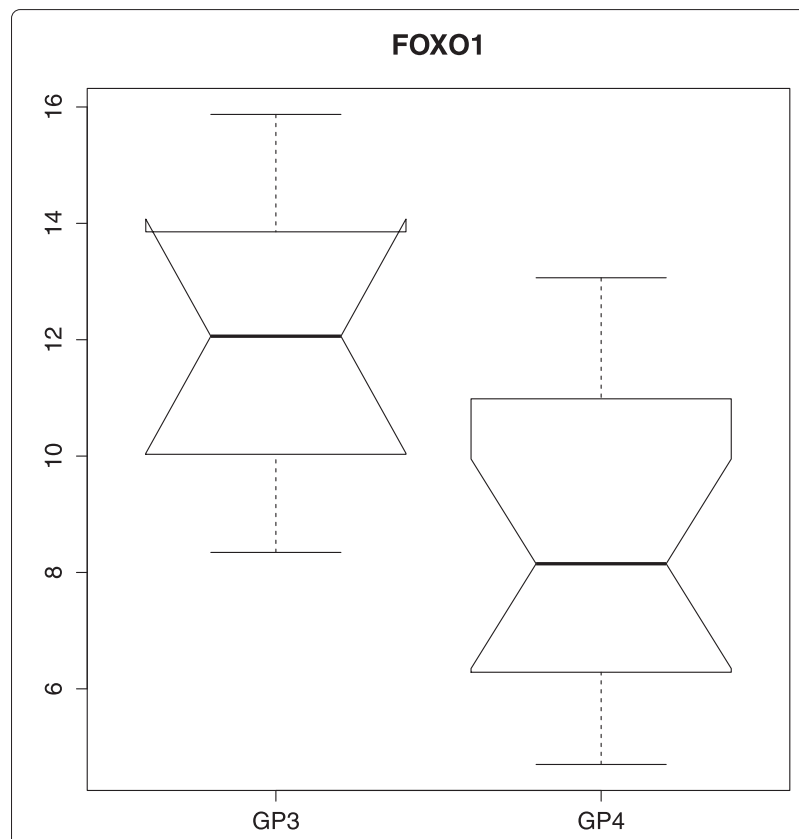

Fig. 1 Boxplot of GP3 vs. GP4 expression for FOXO1. A notched box-and-whisker plot representing the distributions in FOXO1 gene expression between GP3 and GP4. (The solid lines represent the medians, and the notches show the $95 \%$ confidence intervals for the medians. The whiskers represent 1.5 times the inter-quartile ranges.)

The $y$-axis represents $\log _{2}$ expression intensities. The upper GP3 quartile and lower GP4 quartile show minimal overlap, and the median show significant separation, consistent with the results of the MWU test. FOXO1 retained statistical significance even after FDR was applied to the MWU test ( $q=0.0495)$

three pathways were consistently identified: "gluconeogenesis," "hexose catabolic process," and "monosaccharide catabolic process" (Table 2). Analysis of the proteinprotein interactions within the 26-gene network identifies FOXO1 and $A K T 1$ as being primary nodal points based on their number of connecting proteins (Fig. 4). AKT1 and $F O X O 1$ appear to directly affect one another through reciprocal activation or inhibition in a manner that is dependent on the overall metabolic context. Two processes that are not found when one uses only our input genes, but that are consistently reported upon enrichment of five or ten related genes, are the "response to oxygen levels" $\left(p=2.97 \times 10^{-4}\right)$ and "response to hypoxia" $\left(p=3.91 \times 10^{-4}\right)$. To validate these results, the same process was repeated in GeneMANIA with the addition of ten related gene partners. The prevalence of the glucose catabolic pathway was recurrent in both analyses, further substantiating this observation.

\section{TSP analysis}

In order to limit normalization biases resulting from the small number of samples, rank-based TSP of preprocessed raw data was also tested. However, the broad 


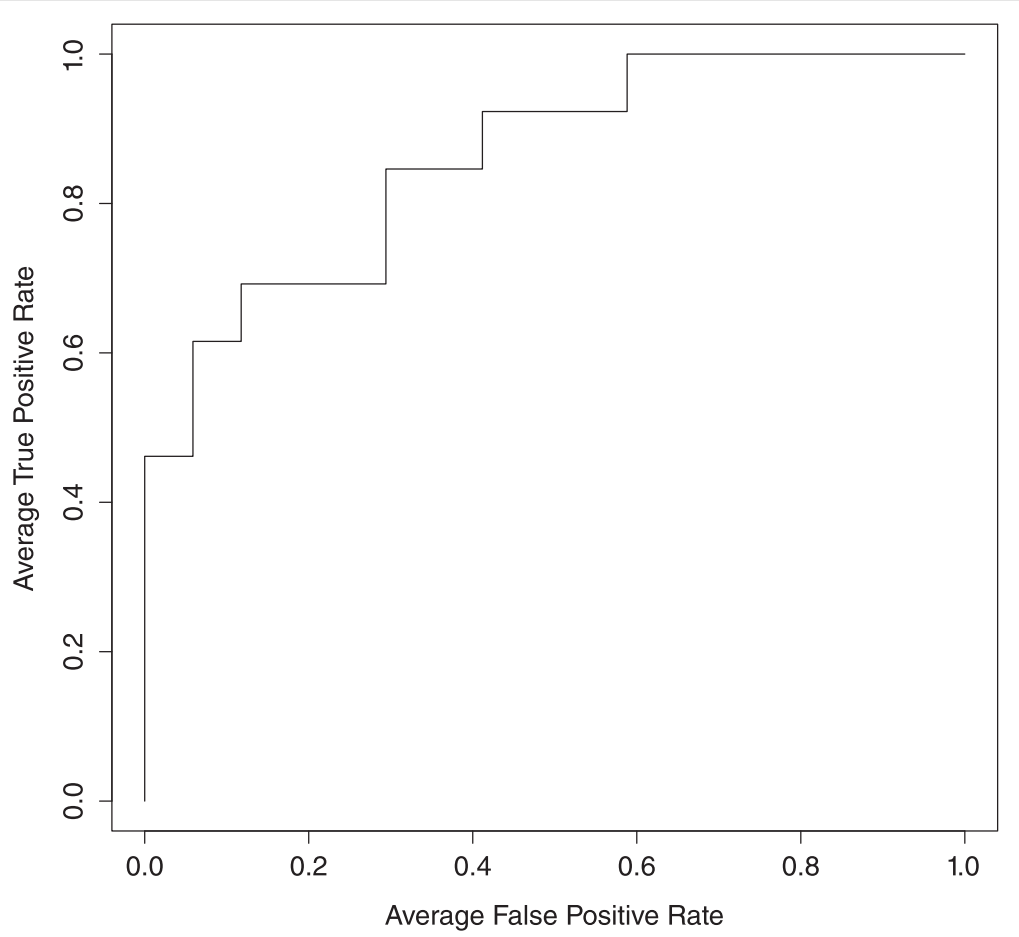

Fig. 2 ROC curve for $F O X O 1$. $A \cup C=0.884$

distribution of negative controls (Additional file 6: Figure S2) indicated that the low intensity readings in our raw dataset were unlikely to be reliable in its present form. Therefore, we chose to apply background correction using the built-in exogenous negative controls. Backgrounds were subtracted from the raw data using the modestly conservative "mean" option in the NanoStringNorm package.

Following mean background correction, and exclusion of genes and samples with $>50 \%$ missing data, four genes and nine samples were excluded. Excluded genes and excluded samples were as follows: HGMCL, IGF2, IL10, NOS2, and TKTL1 and GP3-5, GP3-6, GP3-7, GP3-10, GP3-12, GP3-13, GP3-14, GP3-20, and GP4-12, respectively. Additionally, the single sample (GP4-18) that did not previously pass sample content normalization due to its large scaling factor was also removed, in order for our treatment of the data to remain consistent between the univariate and TSP analyses. The final dataset used for TSP analysis consisted of 13 GP3 and 18 GP4 samples (Additional file 7: Figure S3).

TSP analysis identified a top-scoring pair ATG5/GLUT1 with a score of 0.547 , capable of correctly classifying $24 / 31(77.4 \%)$ samples. To be specific, with the ordering $(\operatorname{Exp}(A T G 5)>\operatorname{Exp}(G L U T 1))$, the scatterplot of Fig. 5 was generated. Permutation testing using 100,000 random assignment classifications of GP3 and GP4 into groups of 13 and 18 generated a distribution of scores (Fig. 6). The low frequency of scores greater than 0.5 indicate that a score of 0.547 for gene pair ATG5/GLUT1 is highly significant $(p=0.0039)$.

\section{Discussion}

The roles of the stromal microenvironment and the RWE have become increasingly noteworthy in the context of cancer progression, and, as such, suggest a potential utility of RWE-associated genes as prognostic biomarkers. Our study has identified an RWE-associated gene, FOXO1 (AUC: 0.884 ), that is significantly differentially expressed between GP3 and GP4 stroma, even after FDR correction, as well as a multivariate top-scoring RWE-associated gene pair, ATG5/GLUT1, whose relative expression can classify GP in $77.4 \%$ of cases. The remaining eight genes, which were significantly differentially expressed in both Welch and MWU tests, but did not reach statistical significance upon FDR correction, are also suggestive of additional classifiers.

If reproducible in subsequent independent cohorts, these RWE-associated biomarkers may have clinical value in risk stratification. The identification of FOXO1 and ATG5/GLUT1 in purified stroma and their differential expression in aggressive vs. indolent prostate cancer samples indicate that it is possible to clinically categorize $\mathrm{PCa}$ in terms of the metabolic responses, namely RWE, that it elicits in the stroma. Additionally, if these gene expression changes extend appreciably beyond the immediate tumor-stroma border, direct sampling of GP4 or GP3 may become less necessary. For example, decreased expression 


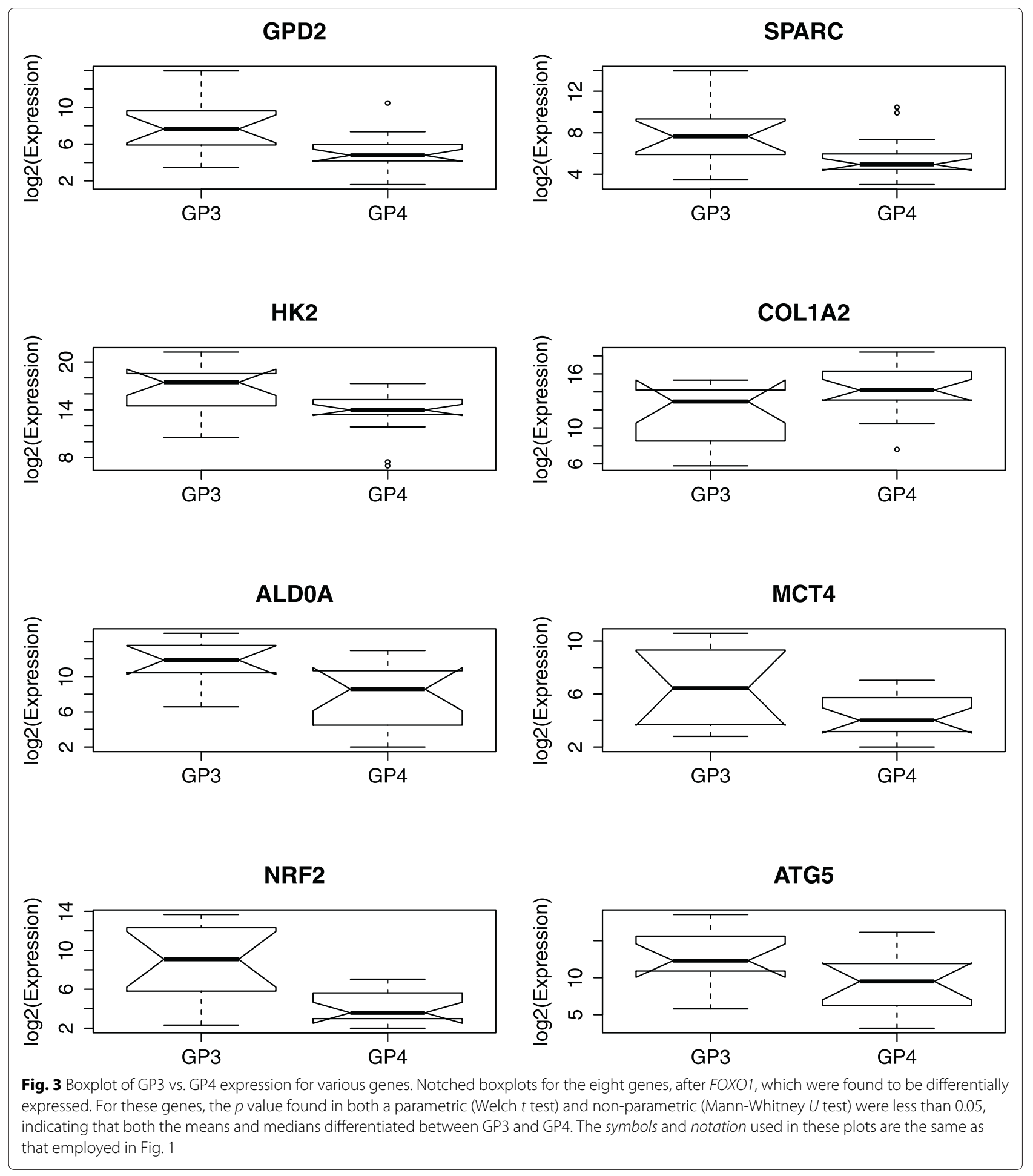

of ATG5 relative to GLUT1 in a biopsy core showing predominantly stroma or only low-grade cancer may indicate the presence of nearby higher-grade cancer that was missed by the biopsy. Evidence to suggest that this type of "field effect" exists within the stroma includes studies that show high autophagic turnover in fibroblasts at a distance of up to $5 \mathrm{~mm}$ away from the cancer [55].
In addition to the identification of two significant classifiers, this study has also provided potential insights into the RWE response as it pertains to different grades of $\mathrm{PCa}$. The expression profiles of low-grade cancer stroma reported here are consistent with Pavlides' model of the RWE, which depend on ROS-induced and HIF1Amediated transcription of genes encoding key glycolytic 
Table 2 Enrichment for $\mathrm{GO}$ biological processes using STRING

\begin{tabular}{llll}
\hline Term & $\begin{array}{l}\text { Number } \\
\text { of genes }\end{array}$ & $q$ \\
\hline Gluconeogenesis & 4 & $5.92 \times 10^{-8}$ & $4.54 \times 10^{-4}$ \\
Hexose catabolic process & 4 & $1.42 \times 10^{-7}$ & $4.54 \times 10^{-4}$ \\
Monosaccharide catabolic process & 4 & $2.33 \times 10^{-7}$ & $4.86 \times 10^{-4}$ \\
\hline
\end{tabular}

enzymes, transporters, and autophagic vesicle assembly factors [35]. The upregulation of MCT4 seen here is consistent with the induction of reciprocal lactate shuttling during RWE [56]. Similarly, the upregulation of genes, $H K 2$, ALDOA, GPD2, and ATG5, which are prerequisites for responses to high glycolytic influx and auto-phagosome formation, respectively, are also consistent with the literature on RWE induction [25, 57]. Interestingly, both of the statistically meaningful classifiers, FOXO1 and ATG5, are directly implicated in the activation of the mitochondrial autophagy response. More specifically, FOXO1 promotes the transcription of the
ATG5 gene [58]. These congruent results, achieved via two different means of data processing, highlight an association between autophagy and GP3 and validate earlier studies that identify mitochondrial dysfunction as the primary mechanism of RWE induction in CAFs [40, 55]. Similarly, since GLUT1 expression in stromal cells reflects their ability to import and metabolize glucose, the relative expression of the TSP genes ATG5 > GLUT1 may indicate that autophagy may be more important to RWE establishment and maintenance than upregulation of glucose intake.

It is, however, noteworthy that other common mitophagy markers such as BNIP3 and MAP1LC3B were not found to be differentially expressed between the Gleason patterns. ATG5 and BNIP3 participate in early and late autophagy response, respectively [59], and therefore the upregulation of ATG5 primarily points towards a role for early autophagosome formation in GP3-induced RWE. HIF1A also showed no significant difference in expression; however, upon enrichment for GO biological

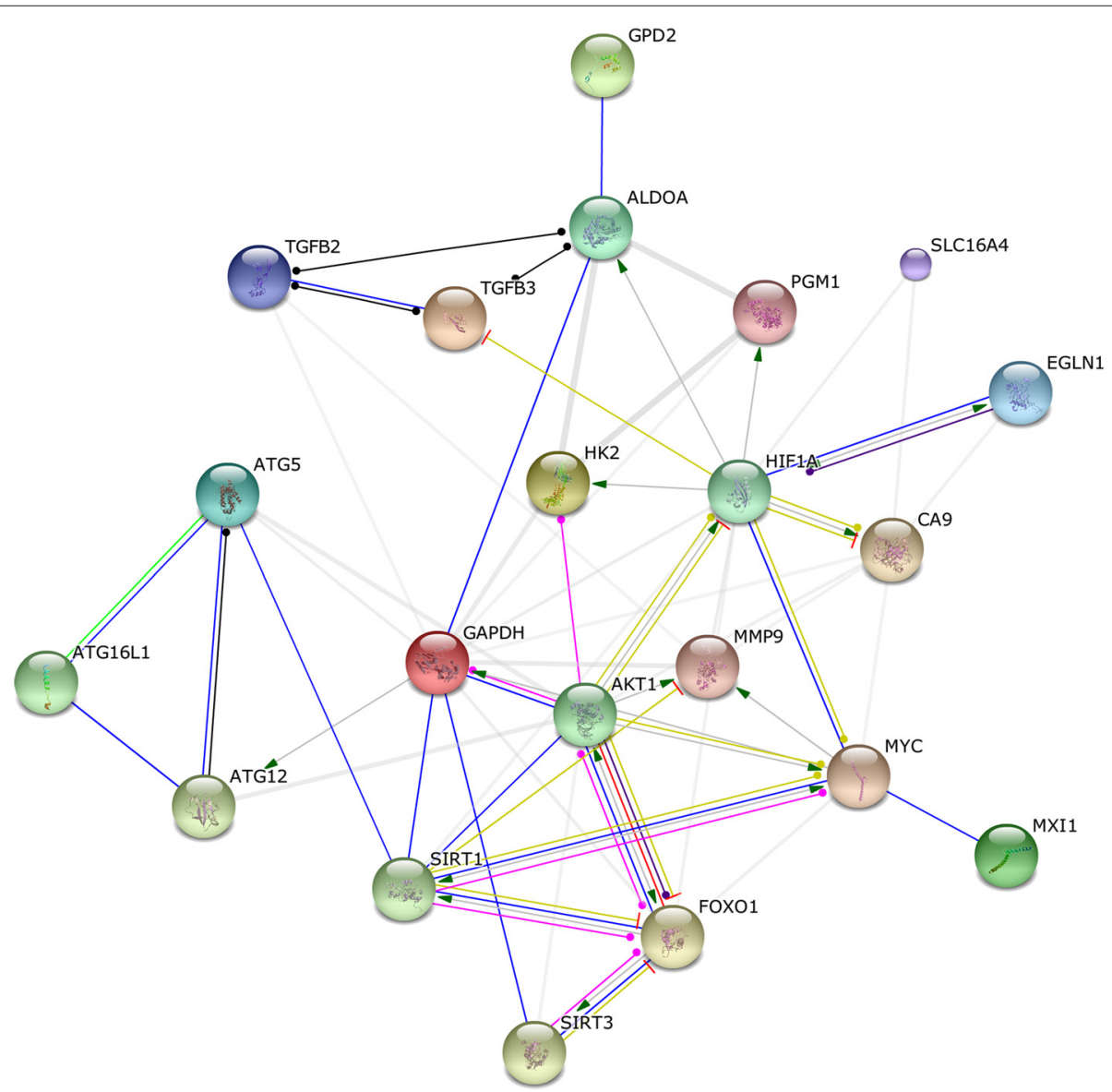

Fig. 4 Protein-protein interactions network generated using STRING. The network is made up of the 17 upregulated genes in GP3 relative to GP4 (FOXO1, GPD2, HK2, MYC, ALDOA, SLC16A4, ATG5, TGFB2, TGFB3, EGLN1, GAPDH, CA9, P4HA1, MXI1, MMP9, and PGM1), plus five enriched genes (ATG16L1, ATG12, AKT1, SIRT1, and SIRT3). The network centers on the primary nodal points: FOXO1, AKT1, MYC, and HIF1A, which show the largest number of reciprocal inhibitory and activating functions with each other as well as with their interacting proteins. Activation (green), inhibition (red), binding (blue), post-translational modification (fuchsia), reaction (black line). Directionality is indicated by the arrow 


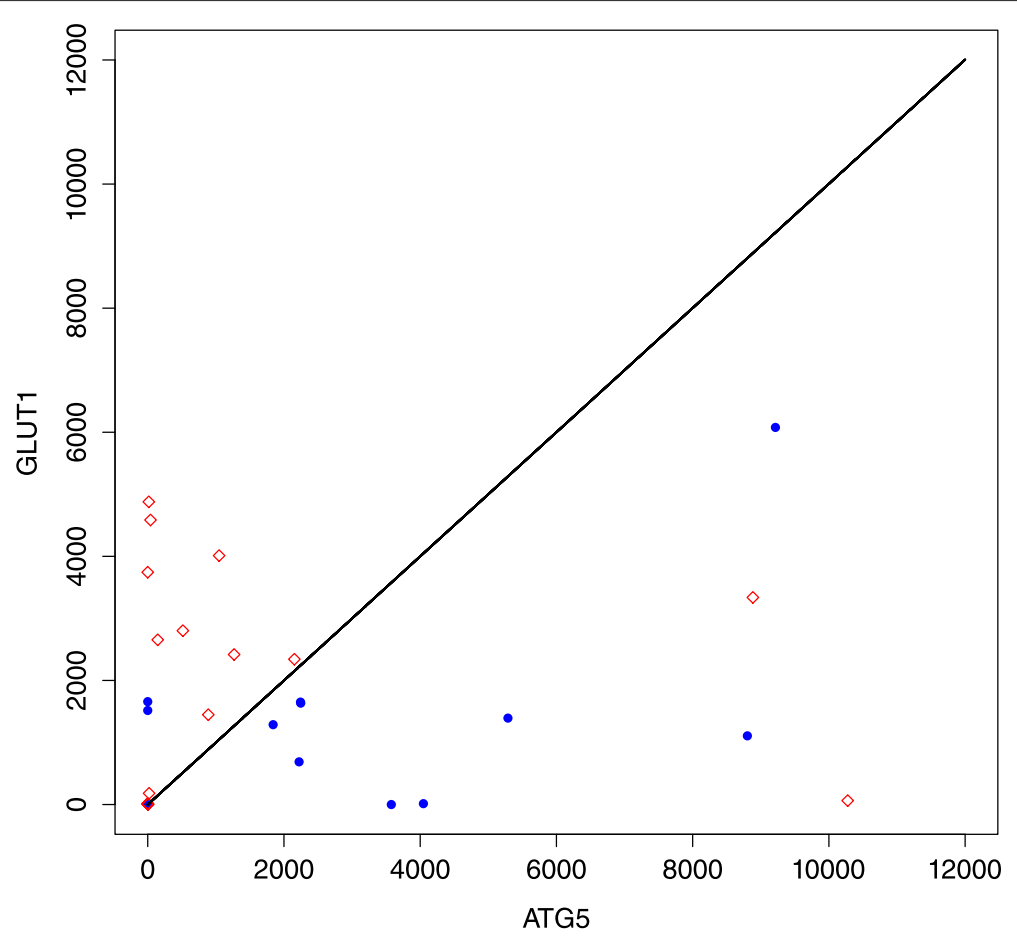

Fig. 5 TSP scatterplot. Scatterplot illustrating the separation of GP3 and GP4 using the expression intensities of gene pair ATG5/GLUT1. With the ordering $\operatorname{Exp}(A T G 5)>\operatorname{Exp}(G L U T 1), 24 / 31$ (77.4\%) samples were correctly classified. One outlier (which agrees with the ordering) has been left out for clarity

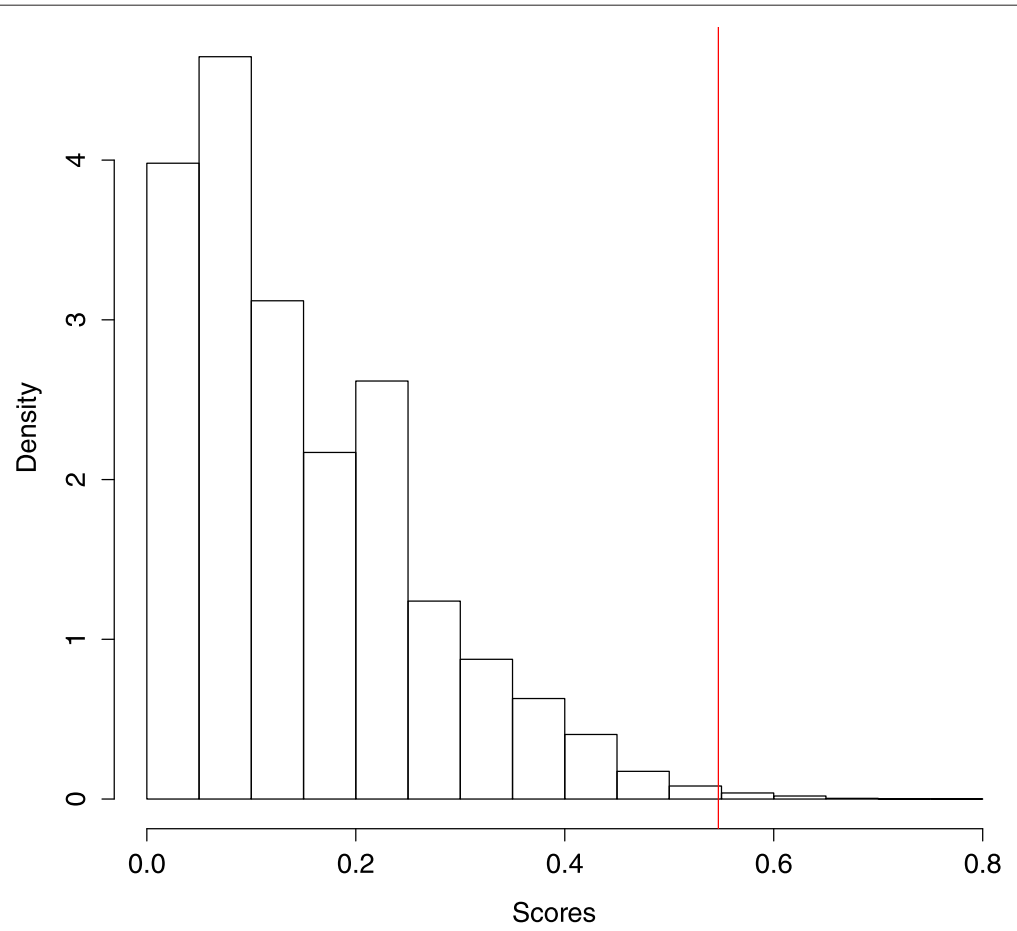

Fig. 6 TSP random classification distribution. Distribution of 100,000 random classification assignment TSP samplings. The low frequency of high scores indicates that the score (0.547—red line) identified for top-scoring gene pair (ATG5/GLUT1) is unlikely to be due to chance $(p=0.0039)$ 
processes, responses to hypoxia and oxygen availability were revealed to be significant. Lastly, we did not observe expression differences in $L D H A$ and $L D H B$, the enzymes involved in conversion of pyruvate to lactate, or in $P D K 1$ which regulates the flux into the tricarboxylic acid cycle. In our context, this may indicate that lactate production may not be as important as lactate export to the RWE in PCa.

Relative to stroma from low-grade cancer, higher-grade stroma exhibited gene expression patterns that indicated a reduced RWE response. These results are in agreement with studies conducted by Koukourakis et al. [60-62] and Rattigan et al. [63] who have reported that CAFs isolated from both lung and colorectal cancers retain their oxidative phosphorylation potential, likely in order to recycle the high volume of lactate secreted by more aggressive tumors. These results also corroborate studies conducted on breast and pancreatic cancers that have reported the reduced importance of RWE in more aggressive disease subtypes such as TNBC and HER2+ [64]. This reduction in RWE response in GP4 relative to GP3 foci leads us to speculate that RWE is perhaps a characteristic of a predominantly proliferative phenotype with rather than invasion and metastasis.

This switch from a predominantly proliferative phenotype in low-grade cancer to an invasive phenotype in higher-grades may explain why genes (COL1A2 and $S P A R C$ ) coding for extracellular matrix (ECM) proteins are independently upregulated in higher-grade stroma but not in low-grade stroma. Cross-linking of CAF-secreted collagens has been shown to promote tumor invasion by increasing ECM stiffness [65]. Binding of secreted COL1A 2 to integrins, such as integrin $\alpha 2 \beta 1$ on the surface of tumors, has also been shown to drive tumor cell migration via the activation of RhoC and PI3 kinase [66]. SPARC has been shown to promote type 1 collagen fibril accumulation and remodelling and to facilitate migration of CAFs by decreasing adhesion to the ECM [67]. Interestingly, SPARC may also play an additional role in controlling metabolism. Its overexpression in the epithelial compartment has been shown to decrease glucose uptake and lactate production [68].

Finally, based on the protein-protein network analysis presented here, we propose that the reduction in RWE that we have observed occurs through the mediation of FOXO1 function. FOXO1 is controlled by competing pathways in the cell, namely activation by ROS [69-71], and inhibition via the insulin-activated PI3K/AKT pathway [72]. Decreased expression of ATG5 in high-grade stroma is consistent with the effects of an activated PI3K/AKT1 pathway. The tendency of GP4 foci to overexpress growth signals such as IGF and EGFR may serve to explain FOXO1 activation in response to GP3 but not GP4 [73]. These results further illustrate the relationship between PCa phenotype and stromal metabolic response.

\section{Conclusions}

Few studies have investigated the use of RWE-associated genes in a prognostic setting. In this study, we have identified two potential classifiers, stromal FOXO1 and stromal ATG5/GLUT1, which have the potential to be used to distinguish between aggressive and indolent forms of $\mathrm{PCa}$. Future directions should include validation of either FOXO1 expression or ATG5/GLUT1 relative expression classifiers in a larger cohort of independent tissue samples. Additionally, we report reduced expression of an RWE gene signature in high-grade stroma, a finding sufficiently robust to achieve significance under two independent statistical analyses. Based on the pathway analysis presented here, this reduction is likely mediated by FOXO1 and AKT1 signalling. Since the role that the RWE and its associated functional pathways play in $\mathrm{PCa}$ growth has to our knowledge not been previously explored, the work reported here indicates the potential benefits of investigating, measuring, and manipulating this pathway in $\mathrm{PCa}$.

\section{Additional files}

Additional file 1: Clinical data. Table S1. Correlations between GP3 and GP4 and secondary clinical characteristics: age, pre-op PSA, \% cancer tissue volume, and pathological stage. (PDF $43.5 \mathrm{~kb}$ )

Additional file 2: Gene/accession numbers. Table S2. RWE code-set accession numbers. (XLSX $46.8 \mathrm{~kb}$ )

Additional file 3: MTE primer sequences. Table S3. MTE primer sequences. (XLSX $45.9 \mathrm{~kb}$ )

Additional file 4: NanoString probe sequences. Table S4. NanoSNanoStringtring probe sequences. (XLSX $42.1 \mathrm{~kb}$ )

Additional file 5: NanoString positive controls. Figure S1. Plot of the $\log _{2}$ raw expression values for six positive controls ranging from a concentration of 128 to $0.128 \mathrm{fM}$. Each box-whisker construct represents one positive control. The settings for the notched box-whisker plots are the same as those in the main text (see caption to Fig. 1). (PDF 5.09 kb)

Additional file 6: NanoString negative controls. Figure S2. Plot of the $\log _{2}$ raw expression values for eight negative controls. The broad distribution is likely due to the fragmentation of small input samples. The broad distribution of the negative controls indicates that the low intensity data is going to be less reliable. Each box-whisker construct represents one negative control. The settings for the notched box-whisker plots are the same as those in the main text (see caption to Fig. 1). (PDF $5.52 \mathrm{~kb}$ )

Additional file 7: NanoString housekeeping genes. Figure S3. Plot of the $\log _{2}$ raw expression values for the five selected housekeeping genes. Distribution of expression values is very broad within each gene; HPRT1 and TUBB have particularly long whisker ranges of 5000. The very broad distribution of these housekeeping genes in stromal tissue made them unamendable to the calculation of normalization factors; therefore normalization factors were calculated using the geometric mean of the top 75 genes within a sample. The settings for the notched box-whisker plots are the same as those in the main text (see caption to Fig. 1). (PDF 5.02 kb) 


\section{Abbreviations}

AUC: area under the curve; CAF: cancer-associated fibroblast; ECM: extracellular matrix; FDR: false discovery rate; FFPE: formalim-fixed paraffin-embedded; FOV: field of view; GO: Gene Ontology; GP: Gleason pattern; GS: Gleason score; MTE: multiplex target enrichment; MWU: Mann-Whitney U; PCa: prostate cancer; ROC: receiver operator characteristics; ROS: reactive oxygen species; TSP: top-scoring pair.

\section{Competing interests}

DMB serves as a paid consultant and has a financial interest in Metamark Genetics. All other authors declare that they have no competing interests.

\section{Authors' contributions}

DB and PCP completed the pathology reviews of the prostate tissues. CD performed the chart reviews and provided the patient data examined by $A D$ RJG helped develop a normalization script, as well as with the univariate statistical analysis of the nCounter expression data. RJG also performed the TSP analysis. IG performed all experiments outlined in this study. Experimental and study design were completed by PCP and IG. As primary author, IG wrote the manuscript, with inputs from RJG, SS, DMB, and PCP. No other co-authors to declare. All authors read and approved the final manuscript.

\section{Acknowledgements}

This study was supported by research grants from the Prostate Cancer Fight Foundation and by Prostate Cancer Canada, Grant \#T2014-01, and proudly funded by the Movember Foundation. PCP was supported by the Ontario Institute for Cancer Research Transformative Pathology Fellowship Program. IG was supported by the Terry Fox Foundation Training Program in Transdisciplinary Cancer Research in partnership with the Canadian Institute of Health Research.

\section{Author details}

${ }^{1}$ Department of Pathology and Molecular Medicine, Queen's University, Kingston, ON, Canada. ${ }^{2}$ Division of Cancer Biology and Genetics, Cancer Research Institute, Queen's University, Kingston, ON, Canada. ${ }^{3}$ Department of Physics, Engineering Physics and Astronomy, Queen's University, Kingston, ON Canada. ${ }^{4}$ Department of Urology, Queen's University, Kingston, ON, Canada. ${ }^{5} \mathrm{NCIC}$ Clinical Trials Group, Queen's University, Kingston, ON, Canada. ${ }^{6}$ Ontario Institute for Cancer Research, Toronto, ON, Canada.

\section{Received: 5 December 2015 Accepted: 29 March 2016 Published online: 05 May 2016}

\section{References}

1. Gleason D, Mellinger G. Prediction of prognosis for prostatic adenocarcinoma by combined histological grading and clinical staging. J Urol. 1974;111:58-64.

2. Stark J, Perner S, Mucci L. Gleason score and lethal prostate cancer: does $3+4=4+3$ ? J Clin Oncol. 2009;27(21):3459-64.

3. Fine S, Epstein J. A contemporary study correlating prostate needle biopsy and radical prostatectomy Gleason score. J Clin Oncol. 2009;179(4):1335-39.

4. Beltran H, Rubin M. New strategies in prostate cancer: translating genomics into clinic. Clin Cancer Res. 2013;19(3):517-22.

5. Berger M, Lawrence M, Demichelis F, Drier Y, Cibulskis K, Sivachenko A, et al. The genomic complexity of primary human prostate cancer. Nat. 2011;470:214-0.

6. Barbieri C, Baca S, Lawrence M, Demichelis F, Blattner M, Theurillat J, et al. Exome sequencing identifies recurrent SPOP, FOXA1 and MED12 mutations in prostate cancer. Nat Genet. 2012;44:685-9.

7. Grasso C, Wu Y, Robinson D, Cao X, Dhanasekaran S, Khan A, et al. The mutational landscape of lethal castration-resistant prostate cancer. Nat. 2012;487:239-43.

8. Taylor B, Schultz N, Hieronymus H, Gopalan A, Xiao Y, Carver B, et al. Integrative genomic profiling of human prostate cancer. Cancer Cell. 2010;18:11-22.

9. Cuzick J, Swanson G, Fisher G, Brothman A, Berney D, et al. Prognostic value of an RNA expression signature derived from cell cycle proliferation genes in patients with prostate cancer: a retrospective study. Lancet Oncol. 2011;12:245-55.
10. Blume-Jensen P, Berman D, Rimm D, Shipitsin M, Putzi M, et al. Development and clinical validation of an in situ biopsy-based multimarker assay for risk stratification in prostate cancer. Clin Canc Res. 2011;21(11):2591-600.

11. Erho N, Crisan A, Vergara I, Mitra A, Ghadessi M, et al. Discovery and validation of a prostate cancer genomic classifier that predicts early metastasis following radical prostatectomy. PLOS One. 2013;8(6): 66855.

12. Klein E, Cooperberg M, Magi-Galluzzi C, Simko J, Falzarano S, Maddala T, et al. A 17-gene assay to predict prostate cancer aggressiveness in the context of Gleason grade, heterogeneity, tumour multifocality, and biopsy undersampling. Eur Urol. 2014;66(3):550-60.

13. True L, Coleman I, Hawley S, Huang C, Gifford D, Coleman R, et al. A molecular correlate to the Gleason grading system for prostate adenocarcinoma. PNAS. 2006;103(29):10991-6.

14. Irshad S, Bansal M, Castillo-Martin M, Zheng T, Aytes A, Wenske S, et al. A molecular signature predictive of indolent prostate cancer. Sci Transl Med. 2013;5(202):202-122.

15. Lapointe J, Li C, Higgins J, van de Rijn M, Bair E, Montgomery K, et al. Gene expression profiling identifies clinically relevant subtypes of prostate cancer. PNAS. 2004;101:811-16.

16. Chung L, Baseman A, Assikis V, Zhau H. Molecular insights into prostate cancer progression: the missing link of tumor microenvironment. J Urol. 2005;173(1):10-20.

17. Kaminski A, Hahne J, Haddouti e-M, Florin A, Wellmann A, Wernert N Tumour-stroma interactions between metastatic prostate cancer cells and fibroblasts. Int J Mol Med. 2006;18(5):941-50.

18. Mueller M, Fusenig N. Friends or foes-bipolar effects of the tumour stroma in cancer. Nat Rev Cancer. 2004;4(11):839-49.

19. Wiseman B, Werb Z. Stromal effects on mammary gland development and breast cancer. Science. 2002;296(5570):1046-49.

20. Olumi A, Grossfeld G, Hayward S, Carroll P, Tisty T, Cunha GR. Carcinoma-associated fibroblasts direct tumor progression of initiated human prostatic epithelium. Cancer Res. 1999;59(19):5002-11.

21. Orimo A, Gupta PB, Sgroi DC, Arenzana-Seisdedos F, Delaunay T, Naeem R, Carey VJ, Richardson AL, Weinberg RA. Stromal fibroblasts present in invasive human breast carcinomas promote tumor growth and angiogenesis through elevated SDF-1/CXCL12 secretion. Cell. 2005;121(3):335-48.

22. DeWever $\mathrm{O}$, Mareel $\mathrm{M}$. Role of tissue stroma in cancer cell invasion. J Pathol. 2004;200(4):429-7.

23. Desmouliere A, Guyot C, Gabbiani G. The stroma reaction myofibroblast: a key player in the control of tumor cell behavior. Int J Dev Biol. 2004;48(5-6):509-17.

24. Giannoni E, Bianchini F, Masieri L, Serni S, Torre E, Calorini L. Reciprocal activation of prostate cancer cells and cancer-associated fibroblasts stimulates epithelial-mesenchymal transition and cancer stemness. Cancer Res. 2010;70(17):6945-56.

25. Martinez-Outschoorn U, Pavlides S, Howell A, Pestell R, Tanowitz H, Sotgia F, et al. Stromal-epithelial metabolic coupling in cancer: integrating autophagy and metabolism in the tumor microenvironment. Int J Biochem Cell Biol. 2011;43(7):1045-51.

26. Sonveaux $\mathrm{P}$, Vegran $\mathrm{F}$, Schroeder $T$, Wergin $M$, Verrax J, Rabbani $Z$, et al Targeting lactate-fueled respiration selectively kills hypoxic tumor cells in mice. J Clin Invest. 2008:118(12):3930-42.

27. Martinez-Outschoorn U, Balliet R, Rivadeneira D, Chiavarina B, Pavlides S, Wang $C$, et al. Oxidative stress in cancer associated fibroblasts drives tumor-stroma co-evolution: a new paradigm for understanding tumor metabolism, the field effect and genomic instability in cancer cells. Cell Cycle. 2010;9:3256-76.

28. Martinez-Outschoorn U, Trimmer C, Lin Z, Whitaker-Menezes D, Chiavarina B, Zhou J, et al. Autophagy in cancer associated fibroblasts promotes tumor cell survival: role of hypoxia, HIF1 induction and $\mathrm{NF} \kappa \beta$ activation in the tumor stromal microenvironment. Cell Cycle. 2010;9: 3515-3.

29. Ma X, Dahiya S, Richardson E, Erlander M, Sgroi D. Gene expression profiling of the tumor microenvironment during breast cancer progression. Breast Cancer Res. 2009;11(1):7.

30. Trujillo K, Jones A, Griffith J, Bisoffi M. Markers of field cancerization: proposed clinical applications in prostate biopsies. Prostate Cancer. 2012;2012:302894. 
31. Walter K, Omura N, Hong S, Griffith M, Goggins M. Pancreatic cancer associated fibroblasts display normal allelotypes. Cancer Biol Ther. 2008;7(6):882-8.

32. Qiu W, Hu M, Sridhar A, Opeskin K, Fox S, Shipitsin M, et al. No evidence of clonal somatic genetic alterations in cancer-associated fibroblasts from human breast and ovarian carcinomas. Nat Genet. 2008;40(5):650-55.

33. Allinen M, Beroukhim R, Cai L, Brennan C, Lahti-Domenici J, Huang H, et al. Molecular characterization of the tumor microenvironment in breast cancer. Cancer Cell. 2004;6(1):17-32.

34. Campbell I, Polyak K, Haviv I. Clonal mutations in the cancer-associated fibroblasts: the case against genetic coevolution. Cancer Res. 2009;69(17): 6765-9.

35. Mohler J, Bahnson R, Boston B, Busby J, D'Amico A, Eastham J, et al. NCCN clinical practice guidelines in oncology: prostate cancer. J Natl Compr Canc Netw. 2010;8(2):162-200.

36. Epstein J, Allsbrook WJ, Amin M, Egevad L. ISUP Grading Committee: The 2005 International Society of Urological Pathology (ISUP) consensus conference on Gleason grading of prostatic carcinoma. Am J Surg Pathol. 2005;29:1228-42.

37. Vander Heiden M, Cantley L, Thompson C. Understanding the Warburg effect: the metabolic requirements of cell proliferation. Science. 2009;324: 1029-33.

38. Pavlides S, Whitaker-Menezes D, Castello-Cros R, Flomenberg N, Witkiewicz A, Frank P, et al. The reverse Warburg effect: aerobic glycolysis in cancer associated fibroblasts and the tumor stroma. Cell cycle. 2009;8: 3984-4001.

39. Gonzalez C, Alvarez S, Ropolo A, Rosenzvit C, Bagnes M, Vaccaro M. Autophagy, Warburg, and Warburg reverse effects in human cancer. Biomed Res Int. 2014;2014:926729.

40. Pavlides S, Tsirigos A, Vera I, Flomenberg N, Frank P, Casimiro M, et al. Transcriptional evidence for the "reverse Warburg effect" in human breast cancer tumor stroma and metastasis: similarities with oxidative stress, inflammation, Alzheimer's disease and "Neuron-Glia Metabolic Coupling". Aging. 2010;2:185-99.

41. Witkiewicz A, Kline J, Queenan M, Brody J, Tsirigos A, Bilal E, et al. Molecular profiling of a lethal tumor microenvironment, as defined by stromal caveolin-1 status in breast cancers. Cell Cycle. 2011;10:1794-809.

42. Sotgia F, Del Galdo F, Casimiro M, Bonuccelli G, Mercier I, Whitaker-Menezes D, et al. Caveolin-1-/- null mammary stromal fibroblasts share characteristics with human breast cancer-associated fibroblasts. Am J Pathol. 2009;174:746-61.

43. Edgar R, Domrachev M, Lash A. Gene Expression Omnibus: NCBI gene expression and hybridization array data repository. Nucleic Acids Res. 2002;30(1):207-10.

44. Ashida S, Orloff M, Bebek G, Zhang L, Zheng P, Peehl D, et al. Integrated analysis reveals critical genomic regions in prostate tumor microenvironment associated with clinicopathologic phenotypes. Clin Cancer Res. 2012;18(6):1578-87.

45. Planche A, Bacac M, Provero P, Fusco C, Delorenzi M, Stehle J, et al. Identification of prognostic molecular features in the reactive stroma of human breast and prostate cancer. PLoS One. 2011;6(5):18640.

46. Dakhova O, Ozen M, Creighton C, Li R, Ayala G, Rowley D, et al. Global gene expression analysis of reactive stroma in prostate cancer. Clin Cancer Res. 2009;15(12):3979-89.

47. Ohl F, Jung M, Xu C, Stephan C, Rabien A, Burkhardt M, et al. Gene expression studies in prostate cancer tissue: which reference gene should be selected for normalization. J Mol Med. 2005;83:1014-24.

48. Waggott D, Chu K, Yin S, Wouters B, Liu F, Boutros P. NanoStringNorm: an extensible R package for the pre-processing of NanoString mRNA and miRNA data. Bioinformatics. 2012;28:1546-48.

49. Benjamini $Y$, Hochberg $Y$. Controlling the false discovery rate: a practical and powerful approach to multiple testing. J R Statist Soc B. 1995;57(1): 289-300.

50. Geman D, D'avignon C, Naiman D, Winslow R. Classifying gene expression profiles from pairwise mRNA comparisons. Stat Appl Genet Mol Biol. 2004;3:1-19.

51. Szklarczyk D, Franceschini A, Wyder S, Forslund K, Heller D, et al. STRING v10: protein-protein interaction networks, integrated over the tree of life. Nucleic Acids Res. 2015;43(Database issue):447-52.

52. Warde-Farley D, Donaldson S, Comes O, Zuberi K, Badrawi R, Chao P, et al. The GeneMANIA prediction server: biological network integration for gene prioritization and predicting gene function. Nucleic Acids Res. 2010;1 (38 Suppl):214-0

53. The Gene Ontology. The Gene Ontology Consortium: going forward. Nucleic Acids Res. 2015;43(Database issue):1049-1056.

54. Newell J, Patrick S, Clawson G. RNA quality analysis of tumor bank specimens and downstream applicability. FASEB J. 2012;26:1034-15.

55. Chaudhri V, Salzler G, Dick S, Buckman M, Sordella R, Karoly E, et al. Metabolic alterations in lung cancer-associated fibroblasts correlated with increased glycolytic metabolism of the tumor. Mol Cancer Res. 2013;11(6):579-92.

56. Fiaschi T, Marini A, Giannoni E, Taddei M, Gandellini P, De Donatis A et al. Reciprocal metabolic reprogramming through lactate shuttle coordinately influences tumor-stroma interplay. Cancer Res. 2012;72: 5130-40.

57. Zhao $X, \mathrm{He} Y$, Chen $\mathrm{H}$. Autophagic tumor stroma: mechanisms and roles in tumor growth and progression. Int J Cancer. 2013;132:1-8.

58. Xu P, Das M, Reilly J, David R. JNK regulates FoxO-dependent autophagy in neurons. Genes \& Dev. 2011;25:310-22.

59. Mehrpour M, Esclatine A, Beau I, Codogno P. Overview of macroautophagy regulation in mammalian cells. Cell Res. 2010;20:748-62.

60. Koukourakis M, Giatromanolaki A, Sivridis E, Gatter K, Harris A. Pyruvate dehydrogenase and pyruvate dehydrogenase kinase expression in non small cell lung cancer and tumor-associated stroma. Neoplasia. $2005 ; 7(1): 1-6$

61. Koukourakis M, Giatromanolaki A, Harris A, Sivridis E. Comparison of metabolic pathways between cancer cells and stromal cells in colorectal carcinomas: a metabolic survival role for tumor-associated stroma. Cancer Res. 2006;66:632-37.

62. Koukourakis M, Giatromanolaki A, Bougioukas G, Sivridis E. Comparison of metabolic pathways between cancer cells and stromal cells in colorectal carcinomas: a metabolic survival role for tumor-associated stroma. Cancer Biol Ther. 2007;6(9):1476-79.

63. Rattigan Y, Patel B, Ackerstaff E, Sukenick G, Koutcher J, Glod J, et al. Lactate is a mediator of metabolic cooperation between stromal carcinoma associated fibroblasts and glycolytic tumor cells in the tumor microenvironment. Exp Cell Res. 2012;318:326-5.

64. Choi J, Kim dH, Jung W, Koo J. Metabolic interaction between cancer cells and stromal cells according to breast cancer molecular subtype. Breast Cancer Res. 2012;15(5):78.

65. Karagiannis G, Poutahidis T, Erdman S, Kirsch R, Riddell R, Diamandis E. Cancer-associated fibroblasts drive the progression of metastasis through both paracrine and mechanical pressure on cancer tissue. Mol Cancer Res. 2012;10:1403-18.

66. Kirkland S. Type I collagen inhibits differentiation and promotes a stem cell-like phenotype in human colorectal carcinoma cells. Br J Cancer. 2009;101(2):320-6.

67. Strandjord T, Madtes D, Weiss D, Sage H. Collagen accumulation is decreased in SPARC-null mice with bleomycin-induced pulmonary fibrosis. AJP-Lung. 1999;277(3):628-35.

68. Hua H, Jiang F, Huang Q, Liao Z, Ding G. Re-sensitization of 5- FU resistance by SPARC through negative regulation of glucose metabolism in hepatocellular carcinoma. Tumour Biol. 2015;26(1):303-13.

69. Nakae J, Kitamura T, Silver D, Accili D. The forkhead transcription factor Foxo1 (Fkhr) confers insulin sensitivity onto glucose-6-phosphatase expression. J Clin Invest. 2001;108(9):1359-67.

70. Chen C, Jeon S, Bhaskar V, Nogueira V, Sundararajan D, Tonic I, et al. FoxOs inhibit mTORC1 and activate Akt by inducing the expression of Sestrin3 and R. Dev Cell. 2010;18(4):592-604.

71. Webb A, Brunet A. FoxO transcription factors: key regulators of cellular quality control. Trends Biochem Sci. 2014;39:159-69.

72. van der Horst A, Burgering B. Stressing the role of FoxO proteins in lifespan and disease. Nat Rev Mol Cell Biol. 2007;8(6):440-50.

73. Skacel M, Ormsby A, Pettay J, Tsiftsakis E, Liou L, Klein E, et al. Aneusomy of chromosones 7, 8, and 17 and amplification of HER-2/neu and epidermal growth factor receptor in Gleason score 7 prostate carcinoma: a differential fluorescent in situ hybridization study of Gleason pattern 3 and 4 using tissue microarray. Hum Pathol. 2001;32(12):1392-7. 